\title{
IMPACT OF WIND GENERATION UNCERTAINTY ON GENERATING CAPACITY ADEQUACY
}

\author{
Aleksandar Dimitrovski and Kevin Tomsovic ${ }^{1}$
}

\begin{abstract}
This paper addresses the issue of generating capacity adequacy in power systems with considerable share of wind generation. It shows on a hypothetical example derived from the WECC system (western US interconnection) how different percentages of wind penetration affect this adequacy. For this purpose, the popular LOLP - loss of load probability (or more accurately, LOLE - loss of load expectation) is used as an objective, probability based, index. It is compared to reserve margin, another popular, deterministic index. Two systems with the same reserve margin or largest unit reserve can have very different risks of being unable to serve the load. It is shown that this discrepancy exacerbates as wind penetration increases.
\end{abstract}

Index Terms - LOLP, reliability, adequacy, power system planning, wind generation, uncertainty.

\section{INTRODUCTION}

$\mathrm{W}$ ind generation has become increasingly popular choice of technology for new capacity additions in power systems worldwide. Several factors have contributed to this trend. Environmental concerns and a constant increase in fossil fuel prices are central to these factors. Moreover, recent legislative moves for green-house gases limitation in the EU and similar laws currently under consideration in the US and other parts of the world make wind economically more competitive with other, traditional sources of energy. There are also other factors, such as, advances in the manufacturing and control technology, which also add to the attraction of wind as a 'green' source of energy.

Unfortunately, more than any any other renewable source, wind is stochastic, and, unlike the other most important renewable source, water, it cannot be stored in its primary form for later use. The operational difficulties that this creates have been recognized for some time now and a number of papers and studies address this topic (for example, [1]). Still, wind uncertainty will impact power systems in a more fundamental manner when wind generation contributes a significant portion of the generation mix.

This paper addresses the issue of generating capacity adequacy in power systems with a considerable share of wind generation. It is one of the scenarios considered in a research project that deals with complex interacting issues in the long

${ }^{1}$ This work was supported in part by the National Science Foundation (NSF) under Grants No. DGE-0108076 and No. EEC 02-24810.

A. Dimitrovski is with the School of EECS, Washington State University, Pullman, WA 99164, USA (e-mail: aleksandar@ieee.org).

K. Tomsovic is with the School of EECS, Washington State University, Pullman, WA 99164, USA (e-mail: kevin_tomsovic@wsu.edu). term investment dynamics of the WECC system (western US interconnection) [2]-[4]. For this purpose, the popular LOLP loss of load probability is used as an objective, probability based, index. Other two popular, deterministic indices are reserve margin and largest unit reserve. It is well known that these indices are inconsistent in terms of risk. Risk in this context is the probability of not being able to serve the load. Two systems with the same reserve margin or largest unit reserve can have very different risks. We investigate how wind penetration in the generation mix affects these relations in a hypothetical example derived from the WECC system.

Wind uncertainty is modeled by adjusting the wind generation units FOR - forced outage rate. This parameter is uncertain itself and adjusting for wind uncertainty makes it even more so. The usual approach to modeling FOR uncertainty is to use a random variable with some probability distribution. Any distribution could be used but, unless it is normal, the result will be analytically intractable and Monte Carlo simulation has to be used. Thus, normal distribution is usually assumed and the LOLP index can also be assumed normally distributed with resultant mean value and variance. Mean value calculation is straightforward and not much different from the 'crisp' case. However, resultant variance calculation is complex and involves finding equivalent covariance matrix. Here, a different approach is used. Instead of assuming probability distribution, we assume an interval of possible values for the FORs. This corresponds to a rectangular possibility distribution of a fuzzy/interval number. The calculation of the resultant LOLP in this case is much simpler.

Another source of uncertainty in generating capacity adequacy assessment is the load profile. The load forecast is always uncertain and this uncertainty can considerably affect both LOLP's expected value and its variance, if probabilities are used. Still, this uncertainty is easier to include in calculations than the FOR uncertainty. Here, we use the same approach for modeling load uncertainty as FOR uncertainty. Load curve is assumed to consist of intervals of possible values. When such an uncertain load model is convolved with the uncertain generation model, the resultant risk index is the uncertain LOLP.

\section{LOLE INDEX}

Loss of load expectation (LOLE) is one of the oldest and probably the most frequently used index in generating capacity adequacy analysis and power system reliability [5]. It 
is usually referred to as LOLP (loss of load probability) although this is a misnomer, as it almost always represents the expected value of unserved load. It can be defined as [6]:

$$
\begin{aligned}
L O L E & =T \sum_{j=1}^{N_{G}} \sum_{i=1}^{N_{L}} P_{i} P_{j} I_{i j} \\
I_{i j} & = \begin{cases}0 & L_{i} \leq G_{j} \\
1 & L_{i}>G_{j}\end{cases}
\end{aligned}
$$

where:

$T$ - the total time length of the load curve;

$L_{i}$ - the $i^{\text {th }}$ load level;

$P_{i}$ - the probability of $L_{i}$ (fraction of total time when the load is equal or bigger than $L_{i}$ );

$N_{L}$ - number of load levels in the discretized load curve;

$G_{j}-$ the $j^{\text {th }}$ generation capacity level;

$P_{j}$ - the probability of $G_{j}$;

$N_{G}$ - number of generation capacity levels in the generation capacity probability table;

Since the load chronology is usually not of interest, it is advantageous to consider the load duration curve (LDC) instead. In this case, the relative LDC becomes the load probability distribution and the above formula describes a convolution of the two random variables of load occurrence and available generation capacity. Depending on the load curve used, the LOLE index holds various meanings. If the individual hourly load values are used, which is the usual meaning of LDC, the value of LOLE is in hours. If only individual daily peak load values are used, arranged in descending order to form a cumulative load model known as daily peak load variation curve (DPLVC), the value of LOLE is in days. Weekly and monthly peak load variation curves can also be defined, although that is not usual.

Calculating the equivalent generation capacity table (the discrete probability distribution of available capacity) is an extensive computational task, especially if there are a large number of units, each with multiple operating states. There are various approximating techniques that can be used in order to simplify and speed up this process [5].

A somewhat different approach is to convolve the load probability distribution with individual generation distributions one unit at a time, instead of building the equivalent generation distribution first and then convolving it with the load curve [7], [8]. To illustrate this, let's assume that we are given an LDC, a set of $n$ generation units with their corresponding capacities, Forced Outage Rates (FORs), and their loading order. For simplicity, we'll assume that each unit $i$ has only two states and can be either fully available or fully unavailable with probabilities $p_{i}=1-\mathrm{FOR}_{i}$ and $q_{i}=\mathrm{FOR}_{i}$, respectively. The production of the $k+1$ unit in the order, $W_{k+1}$, depends on whether the previous unit, $k$, is available or not:

$$
W_{k+1}=\int_{\sum_{i=1}^{k} P_{i}}^{\sum_{i=1}^{k} P_{i}+P_{k+1}} D_{k}(P) d P \cdot p_{k}+\int_{\sum_{i=1}^{k-1} P_{i}}^{\sum_{i=1}^{k-1} P_{i}+P_{k+1}} D_{k}(P) d P \cdot q_{k}
$$

where $D_{k}(P)$ is the equivalent inverse LDC, obtained after convolving the $k^{\text {th }}$ unit. The integrals in the above expression can be combined if they have the same lower and upper limits. For that purpose, the integrand of the second integral is shifted to the right along the $x$-axis for the capacity of $k^{\text {th }}$ unit, $P_{k}$. The result is:

$$
W_{k+1}=\int_{\sum_{i=1}^{k} P_{i}}^{k} D_{k}(P) d P \cdot p_{k}+\int_{\sum_{i=1}^{k} P_{i}}^{\sum_{i=1}^{k} P_{i}+P_{k+1}} D_{k}\left(P-P_{k}\right) d P \cdot q_{k} .
$$

From (3), we finally get:

$$
W_{k+1}=\int_{\sum_{i=1}^{k} P_{i}}^{k} D_{k+1}(P) d P
$$

where:

$$
D_{k+1}(P)=D_{k}(P) \cdot p_{k}+D_{k}\left(P-P_{k}\right) \cdot q_{k}
$$

is the equivalent inverse LDC, obtained after convolving the unit $k+1$. It accounts for the actual load and the forced outages of all units up to $k$.

This process is illustrated on Figure 1. The curve after convolving unit $k, D_{k+1}$, is obtained as a sum of the curve before convolving unit $k, D_{k}$, multiplied by unit $k$ availability, $p_{k}$, and the shifted $D_{k}\left(P-P_{k}\right)$, multiplied by unit $k$ unavailability, $q_{k}$. In this particular case, $P_{k}$ is $200 \mathrm{MW}$ and $q_{k}$ is 0.2 .

Equation (5) gives the recursive formula for calculating the equivalent inverse LDC. At the beginning, $D_{0}$ is the original inverse LDC, obtained from the load profile. Proceeding in loading order, each unit's equivalent curve and production can then be calculated. After convolving all $n$ units, the final curve $D_{n+1}$ contains the information about the LOLE and the expected energy not served (EENS, also known as expected unserved energy, EUE):

$$
\begin{aligned}
& \text { LOLE }=D_{n+1}\left(\sum_{i=1}^{n} P_{i}\right) \\
& \text { EENS }=\int_{\sum_{i=1}^{n} P_{i}}^{\infty} D_{n+1}(P) d P
\end{aligned}
$$




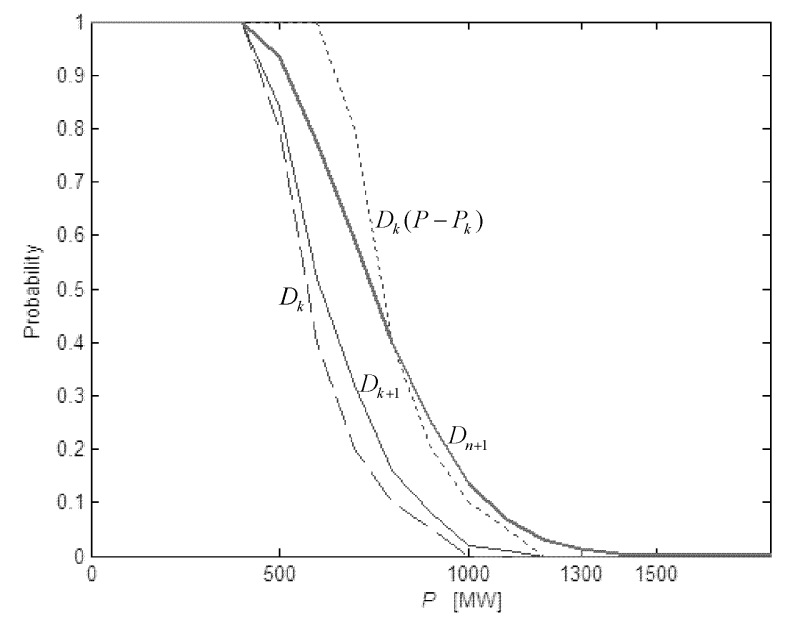

Figure 1. Convolving equivalent inverse LDCs in the process of LOLE and EENS calculation. Curve before convolving unit $k, D_{k}-$ dashed line; shifted $D_{k}$ - dotted line; Curve after convolving unit $k, D_{k+1}-$ solid line; Final convolved curve $D_{n+1}-$ thick solid line; LOLP $=D_{n+1}(1300)=0.0123$.

In the example shown in Figure 1, the final curve is obtained after convolving 9 units with total installed capacity of $1300 \mathrm{MW}$. Thus, the LOLE in this particular case is $D_{n+1}(1300)=0.0123$. The EENS is the area under $D_{n+1}$, starting from 1300 , and is equal to $1.2 \mathrm{MWh}$.

\section{WINDPARK MODELING}

The power of wind is harnessed in windparks that can contain hundreds of individual units (a combination of turbine, generator, tower and other supporting equipment). The installed capacity of each unit is typically between 0.5 MW and $2 \mathrm{MW}$. The large number of units in one "wind plant" distinguishes this type of power plant from conventional thermal power plants. In addition, the FOR of wind units is typically several times less than that of thermal units. For the former, the FOR is somewhere around $1 \%$ or $2 \%$, while for the latter the FOR is on average around $10 \%$. This means that, unlike thermal plants, a windpark has nearly zero probability of being completely outaged and is always ready for service, at least to some extent.

For example, let's consider a windpark with 100 identical units each with $1 \mathrm{MW}$ of installed capacity and $1 \%$ FOR. It's easy to show by using binomial distribution that the probability of having in service less than $76 \mathrm{MW}$ (i.e. $76 \%$ of its installed capacity) is smaller than $10^{-4}$. This is 1000 times less than the probability of a thermal unit with $10 \%$ FOR being completely shut down. If the FOR of wind units is $2 \%$ then the threshold with $10^{-4}$ probability drops down by not much to $63 \mathrm{MW}$.

Therefore, the unit FOR is much less of an issue with wind generation than it is with thermal technologies. It can be taken into account simply by appropriately adjusting the installed capacity of the entire windpark. Here, we should make two important comments. First, we neglect network issues in this paper, following tradition in generating adequacy analysis but, of course, an entire windpark can occasionally be in outage due to a network failure. Second, ready for service does not mean that the windpark will be in service. That depends on the availability of the wind.

The last comment points out the most important thing in defining the availability of a windpark. Due to wind stochasticity the production from a windpark is stochastic and intermittent. Different studies [1] show that the average capacity factor from a windpark is somewhere around $1 / 3$. The capacity factor is defined as the ratio of the time ready for service and the actual time in service. An equivalent FOR for the entire windpark, equal to 1 - capacity factor, is used to model its stochastic production.

\section{PARAmeter UnCertainty}

The LOLE and EENS indices are probabilistic in their nature but, thus far, we have assumed that the load profile and units FOR are known with complete certainty (i.e., with $100 \%$ probability). In practice, this assumption, of course, is never true. Even for the present time these parameters are never precisely known and their uncertainty only grows as we project them further in the future. As said previously, the usual approach to take these uncertainties in account is to use random variables with some probability distributions, usually Gaussian [5]. The use of a normal distribution makes the results analytically tractable. In every other case, Monte Carlo simulation is the only feasible approach.

Here, we propose the use of interval numbers to model these uncertainties. There are two reasons to pursue this approach. First, we argue that using a probability distribution to model a future system's parameters violates the underlying assumption in probability theory of repetition of events. The future system will most likely be different and operate under different conditions in different environment. Second, interval arithmetic is almost always more straightforward and much simpler than dealing with random variables, even normal ones.

Extending the calculation of LOLE and EENS to interval numbers is simple. The recursive formula for calculating the equivalent inverse LDC in (5) is to be applied according to the rules of interval arithmetic. The LDC in this case is an interval value function, i.e., at any given time the load is described by not just one, but by an interval of values. Thus, $D_{k}$ is a "thick" curve, has multiple values along the $\mathrm{x}$-axis for the same probability. This, in turn, renders $D_{k+1}$ and all subsequent curves "thick" as well. Finally, applying expressions (6) and (7) on a "thick" $D_{n+1}$ results in interval values for LOLE and EENS.

Since interval numbers are just a special case of fuzzy numbers, which can be seen as lumped and nested intervals, we can easily extend further calculation of LOLE and EENS to the fuzzy case. Thus, we can calculate these indices with different possibilities representing different degrees of belief. This opens an interesting perspective in the planning process where one can calculate possibilities of different risks for various alternatives and weigh outcomes of different decisions accordingly. 


\section{CAse STUdies}

Let's show a hypothetical example derived from the WECC system how different percentages of wind penetration affect generating capacity adequacy. The current thermal generation in this system consists of a large number of units utilizing different technologies. We will assume that they can be classified in 5 categories with maximum available capacities given in Table I. The maximum available capacity, $P_{\max }$, is obtained from the total installed capacity reduced by the capacity on scheduled maintenance. Also, we will define an average unit capacity for each category, $P_{\text {avg }}$, and we will assume that all units within a category are the same and have that capacity. These data are also given in Table I.

TABLE I WECC THERMAL GENERATION CATEGORIES

\begin{tabular}{c|ccccc}
\hline $\begin{array}{c}\text { Thermal } \\
\text { Technology }\end{array}$ & Nuclear & Coal & $\begin{array}{c}\text { Comb. } \\
\text { Cycle }\end{array}$ & $\begin{array}{c}\text { Gas } \\
\text { Steam }\end{array}$ & $\begin{array}{c}\text { Comb. } \\
\text { Turbine }\end{array}$ \\
\hline$P_{\max }[\mathrm{GW}]$ & 7.5 & 29.6 & 53.1 & 20.3 & 19.2 \\
$P_{\text {avg }}[\mathrm{MW}]$ & 750 & 400 & 250 & 125 & 125 \\
\hline
\end{tabular}

On the demand side, Figure 2 shows "thermal load" profile for a summer day. This load is obtained from data for the total demand in the system reduced by the production from hydro units. In this particular case, hydro units cover $29 \%$ of the peak demand and $25.4 \%$ of the daily energy.

We do not include the hydro portion of the system here for the sake of simplicity as this is a fairly complex problem by itself. Hydro units availability depend not only on equipment outages but also on the reservoir head and, therefore, on the level of reservoir depletion. The latter is a stochastic variable with seasonal variation and it can have a dominant effect on the unit availability, more than equipment outages which, on the other hand, are usually much less frequent in hydro units

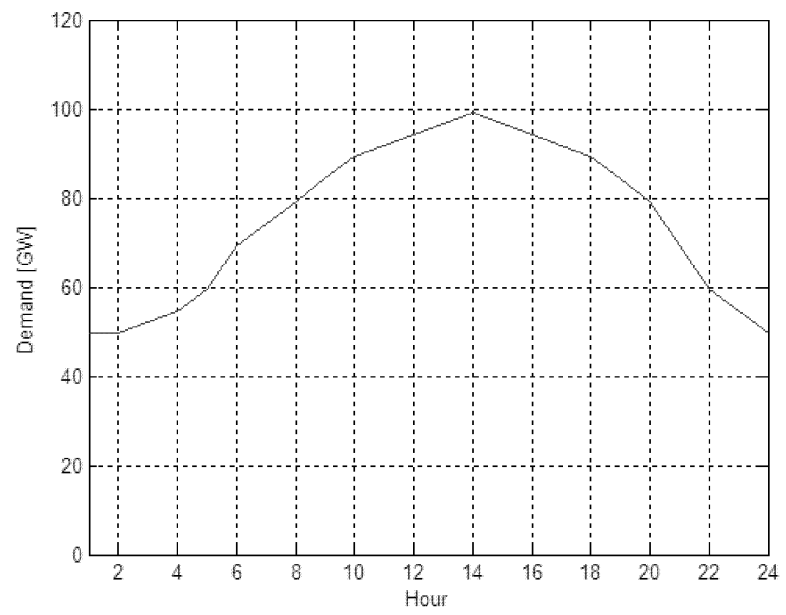

Figure 2. Thermal load profile in the WECC system for a summer day.

than in thermal. The usual approach is to deal with hydro production as a separate subproblem, obtain representative samples from its probability distribution with corresponding probabilities, and then solve the main problem with known hydro by using conditional probabilities.
With hydro portion of the system left aside, we'll introduce another category for wind units with average unit size of 100 MW. As explained in the previous section on windpark modeling, the average unit here represents an entire windpark not an individual unit. We assume an equivalent FOR that accounts for windpark's stochastic production of $2 / 3$. Thus, its capacity factor is $1 / 3$.

Let's now increase the percentage of the wind generation in the total thermal generation mix of the system from $0 \%$ to $15 \%$. In order to keep the same reserve margin, we proportionally increase the load at the same time. The results are displayed in Figure 3. The system LOLE index increases from $8 \cdot 10^{-18}$ to $8.7 \cdot 10^{-6}$ with increased wind penetration in the generation mix. Although the change is significant the final value still seems rather small. This can be attributed to the very large number of units in the system. Thus, the probability of significant outage simultaneously involving a large number of units is very small. However, this small number can be misleading as we show next.

Let's compare the results with the established planning reference value for LOLE of 1 day in 10 years. In other words, the target planning LOLE is usually set at $1 / 3650=$ $2.7 \cdot 10^{-4}$. This value is calculated on the basis of the daily peak load variation curve. In our case, since we have data for only one day, we will calculate the single probability of not meeting the peak load in this day. Note that in this case it is correct to refer to this value as LOLP.

The results of these calculations are shown on Figure 4. The LOLP index for the peak summer load increases from $1.8 \cdot 10^{-16}$ to $2 \cdot 10^{-4}$ with increased wind generation from $0 \%$ to $15 \%$. If these results are extended to a 10 year period then the last value corresponds to 0.735 , or 268 days a year the system

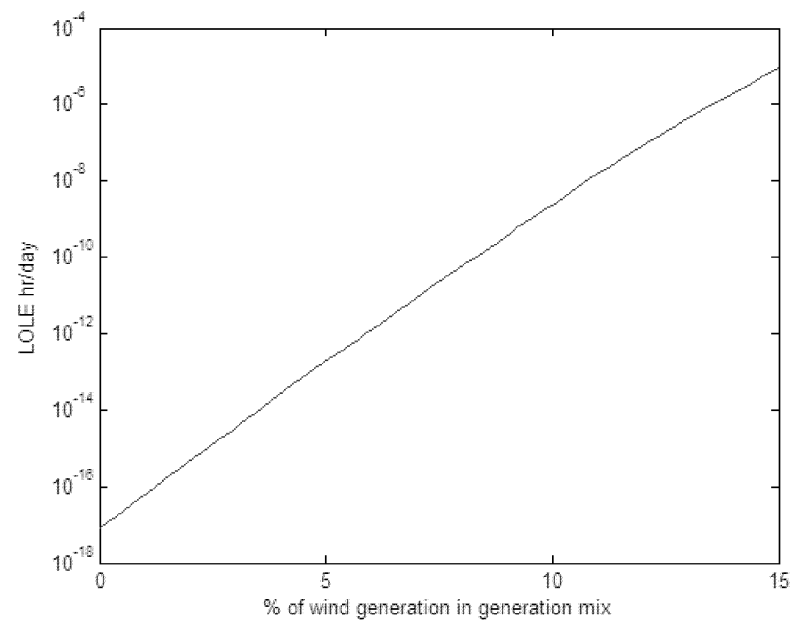

Figure 3. Dependence of the WECC LOLE index on the wind generation in the generation mix - constant reserve margin of $30.9 \%, 10 \%$ FOR for all thermal units. 




Figure 4. Dependence of the WECC LOLP index for a single peak summer load on the wind generation in the generation mix - constant reserve margin of $30.9 \%, 10 \%$ FOR for all thermal units.

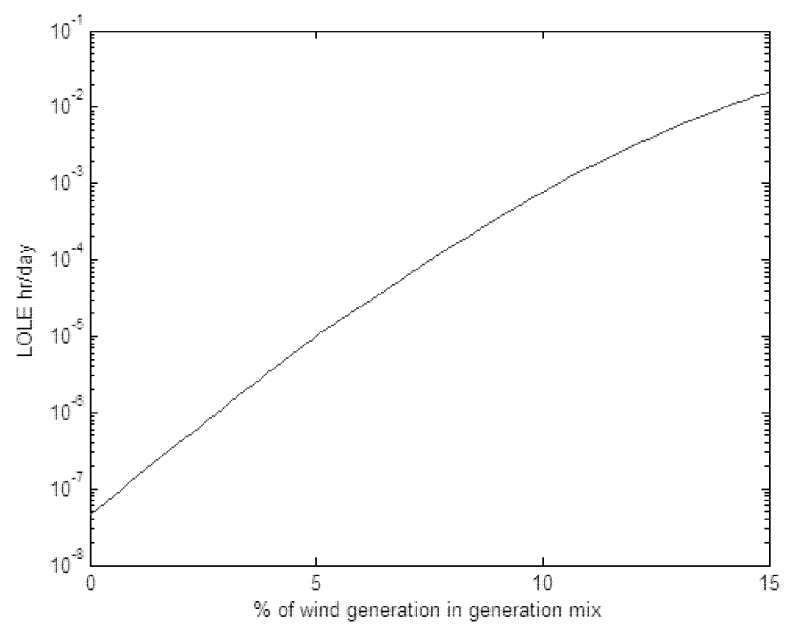

Figure 5. Dependence of the WECC LOLE index on the wind generation in the total generation mix - constant reserve margin of $30.9 \%, 15 \%$ FOR for all thermal units.

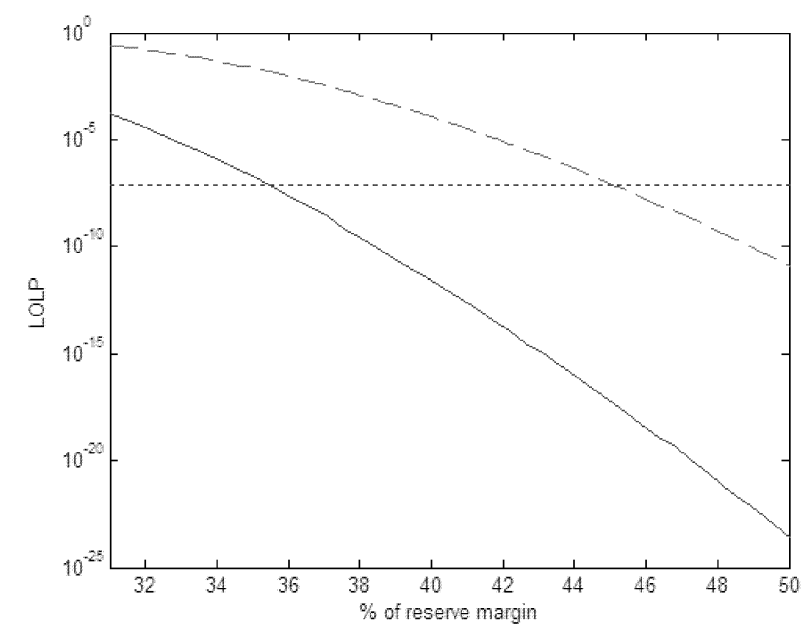

Figure 6. Required reserve margin in the WECC system to meet the target LOLE of 0.1 day/year (dotted line), with $15 \%$ wind generation, for two different values of thermal units FOR: 10\% - solid line; $15 \%$ - dashed line. will not be able to meet its peak load! This is by no means acceptable. True, this result is exaggerated as peak daily loads throughout the year will be smaller than the peak summer day load. However, the real LOLE will still have the same order of magnitude as the result just calculated.

In order to confirm the dramatic effect unit availabilities can have on system adequacy and further emphasize the impact of wind generation in such case, let's assume that all thermal units in the system have FOR of $15 \%$. The results for this case are displayed on Figure 5. The system LOLE index increases from $4.6 \cdot 10^{-8}$ to an extraordinary $0.0159 \mathrm{hr} /$ day with an increase of wind generation from $0 \%$ to $15 \%$.

All the results presented so far show that the system can not be left with the same reserve margin as the percentage of wind generation increases, or its reliability will suffer greatly. The more appropriate analysis is to determine the required reserve margin in order meet the reference LOLE of 1 day in 10 years, when the wind generation percentage is kept fixed at $15 \%$ of the total generation mix. Again, in the first approximation, we'll assume that all days are equal. This sets the target LOLP at $7.5 \cdot 10^{-8}$. The results of these calculations for two different values of FOR are shown on Figure 6. The required reserve margins are $45.1 \%$ and $35.4 \%$, for the $15 \%$ and $10 \%$ values of FORs, respectively. If one takes into account the capacity from hydro units than the actual values are $32 \%$ and $25.1 \%$, respectively.

If there is no wind generation in the system, the target LOLP will be satisfied for reserve margins at $32.9 \%$ and $23.2 \%$, for the $15 \%$ and $10 \%$ values of FORs, respectively. Again, taking into account the hydro capacity, the real values are $23.3 \%$ and $16.5 \%$, respectively. Thus with $15 \%$ wind generation, an additional $8.7 \%$ ( $8.6 \%$ for $10 \%$ FOR) of reserve margin is required to cover the uncertain $15 \%$ of wind. The amount of additional reserve is more than half the wind generation. If the system were purely thermal then an additional $80 \%$ reserve would be required. All these values, of course, depend on the specific case assumptions, units and system parameters, and cannot be strictly generalized.

Let's now see how parameter uncertainty affects adequacy of the system. Consider the same case as in Figure 3, but with $\pm 5 \%$ variation in the load profile and unit FORs. In other words, one assumes the load to be defined as a set of intervals with upper and lower bounds at $95 \%$ and $105 \%$ of the values shown in Figure 2, respectively. Thermal units FORs are given with the interval number $[9.5 \%, 10.5 \%]$ and wind units FORs with [63.3\%, 70\%]. Figure 7 shows the upper and lower boundary for the resultant LOLE in this case. It also shows the crisp case result from Figure 3, which falls between the two boundaries. It can be seen that even a small uncertainty in parameters makes a significant difference. This is especially true for small values of LOLE at the extreme end of the $D_{n+1}$ curve. The range of values gradually shrinks as the LOLE increases and moves away from the tip of $D_{n+1}$. 


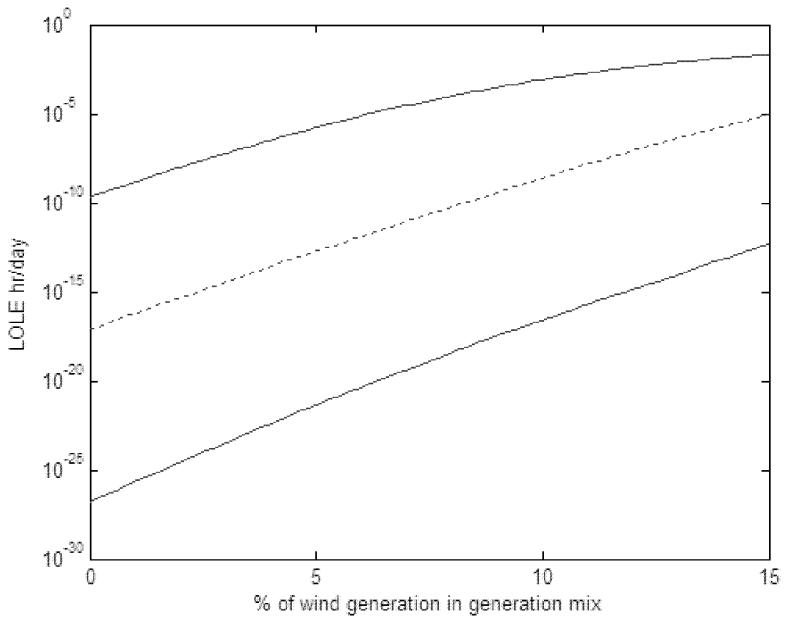

Figure 7. Upper and lower bound of the WECC LOLE index from Figure 3 with $\pm 5 \%$ variation in load profile and unit FORs - reserve margin of $30.9 \%$, $15 \%$ wind penetration.

\section{CONCLUSIONS}

A significant increase in wind generation in modern power systems will have a profound impact on their operation and planning. This paper addresses the fundamental problem of generating capacity adequacy in expansion planning. An approach is used where the entire windpark is modeled as a single unit with an equivalent FOR that accounts for wind uncertainty.

It is shown on a hypothetical, but realistic example, that a considerable amount of additional, non-wind based, sources is needed to counteract wind stochasticity and maintain an acceptable level of risk. It is confirmed again that two systems with the same reserve margin or largest unit reserve can have very different risks levels. This discrepancy grows with the wind penetration.

The amount of additional reserves needed can range anywhere from more than half to more than $80 \%$ of the wind generation. These values, of course, depend on the particular case, units and system parameters. The tendency, however, is obvious and calls for careful planning of additional resources whenever wind units are to be a significant part of the system mix.
Finally, the use of interval numbers is proposed for modeling future system parameter uncertainty. It is a simpler and, the authors believe a more appropriate approach. It can also be easily extended to the use of fuzzy numbers, which allows for a more intuitive approach to decision making under uncertainty in the expansion planning process.

\section{REFERENCES}

[1] UWIG Operating Impacts Study, online at: http://www.uwig.org

[2] A. Dimitrovski, K.Tomsovic, A. Ford, "Comprehensive Long Term Modeling of the Dynamics of Investment and Network Planning in Electric Power Systems" submitted to a special issue of the International Journal of Critical Infrastructures.

[3] A. Ford, K. Vogstad and H. Flynn, "Simulating Price Patterns for Tradable Green Certificates to Promote Electricity Generation from Wind', to be published in Energy Policy.

[4] A. Dimitrovski, K. Tomsovic, "Market Feedback for Bounding Future Uncertainties in Power System Planning", ISAP 2005 Conference, Washington DC, November 6-10.

[5] R. Billinton and R.N. Allan, Reliability Evaluation of Power Systems, New York: Plenum Press, 1996.

[6] W. Li, Risk Assessment of Power Systems - Models, Methods, and Applications, New York: IEEE Press, 2005.

[7] -, Expansion Planning for Electrical Generating Systems - A Guidebook, International Atomic Energy Agency, Vienna, Austria, 1984.

[8] A. Wood and B. Wollenberg, Power Generation, Operation, and Control, New York: John Wiley, 1996.

\section{BIOGRAPHIES}

Aleksandar Dimitrovski received his $\mathrm{BS}$ and $\mathrm{Ph} . \mathrm{D}$. both in power engineering from University "Sv. Kiril i Metodij" in Skopje, Macedonia, and MS in application of computer sciences from University of Zagreb, Croatia. He was an Assistant Professor in power systems at University "Sv. Kiril i Metodij" from 2000-2004. He is currently a visiting professor at Washington State University. His interest lies in advanced computing techniques in power system analysis.

Kevin Tomsovic received the BS from Michigan Tech. University, Houghton, in 1982, and the MS and Ph.D. degrees from University of Washington, Seattle, in 1984 and 1987, respectively, all in Electrical Engineering. He is currently Program Director in the ECS Division of the Engineering Directorate at National Science Foundation and a Professor in the School of EECS at Washington State University. Visiting university positions have included Boston University, National Cheng Kung University, National Sun Yat-Sen University and the Royal Institute of Technology in Stockholm. He held the Advanced Technology for Electrical Energy Chair at Kumamoto University in Japan from 1999-2000. 